\title{
CARACTERIZAÇÃO ANATÔMICA MACROSCÓPICA DA MADEIRA DE QUATRO ESPÉCIES DE LECYTHIDACEAE
}

\author{
Pamella Carolline Marques dos Reis Reis ${ }^{{ }^{*}}$ \& Leonardo Pequeno Reis ${ }^{1}$ \\ ${ }^{1}$ Doutorando (a) do Programa de Pós-graduação em Ciência Florestal, \\ Departamento de Engenharia Florestal, Universidade Federal de Viçosa, Viçosa, \\ MG, Brasil. (pamella.reis@ufv.br) \\ Recebido em: 03/10/2016 - Aprovado em: 21/11/2016 - Publicado em: 05/12/2016 \\ DOI: 10.18677/EnciBio_2016B_016
}

\begin{abstract}
RESUMO
As espécies de Lecythidaceae possuem distribuição pantropical, concentrada na região neotropical, inclui cerca de 25 gêneros e 300 espécies. As espécies dessa família possuem elevado potencial econômico na região amazônica, tendo mais de 28 espécies comercializadas em 2012 no estado do Pará. Objetivou-se com este trabalho caracterizar macroscopicamente, espécies amazônicas, conhecidas popularmente como Mata-matá, disponíveis na xiloteca do Museu Paraense Emílio Goeldi, para contribuir para o conhecimento científico de espécies amazônicas e fornecer informações para a industria e/ou pessoas que lidam com essa matériaprima. Todas as espécies analisadas apresentaram parênquima axial paratraqueal vasicêntrico e em linhas. Poros predominantemente solitários, difusos, tendendo a diagonal. Raios não estratificados contrastados em Eschweilera juruensis R. Knuth e Eschweilera ovata (Cambess.) Miers e pouco contrastados em Eschweilera amazonica R. Knuth e Eschweilera coriacea (DC.) S.A. Mori. Apenas Eschweilera ovata apresentou camadas de crescimento individualizadas por zona fibrosa tangenciais mais escuras, as demais espécies apresentaram camadas de crescimento indistintas.
\end{abstract}

PALAVRAS-CHAVE: Anatomia do lenho, espécies florestais, Matá-matá

\section{ANATOMIC MACROSCOPIC CHARACTERIZATION OF WOOD FROM FOUR LECYTHIDACEAE SPECIES}

\begin{abstract}
The species of Lecythidaceae has a pantropical distribution, concentrated in the Neotropics. The species of this family have high economic potential in the Amazon region, with over 28 species commercialized in 2012 in the state of Pará. The objective of this study was to macroscopically characterize species popularly known as Mata mata, available in xilotheques of Museu Paraense Emilio Goeldi and Embrapa Amazônia Oriental, to contribute to the scientific knowledge of Amazon species and provide information for the industry and/or people who deal with this raw material. All species analyzed showed paratracheal vasicentric axial parenchyma and in lines. Predominantly solitary, diffuse, tending to diagonal pores. Non-stratified
\end{abstract}


rays contrasted in Eschweilera juruensis Kunt and Eschweilera ovata Mart. and little contrasted in Eschweilera Amazon Knuth and Eschweilera coriacea Martius. Only Eschweilera ovata Mart. showed individual growth layers by darker tangential fibrous zone, the other species showed indistinct growth layers.

KEYWORDS: Anatomy of wood, forest species, Mata mata.

\section{INTRODUÇÃO}

Espécies de madeira tropicais ocupam um lugar importante na indústria da construção. Devido às suas propriedades únicas e estética, que são preferidas para a fabricação de uma variedade de produtos, incluindo móveis, armários, arquitectura de madeira, convés e molduras. Dentre estas inclui-se as espécies de Lecythidaceae (DADZIE et al., 2016).

A Lecythidaceae possui distribuição pantropical, concentrada na região neotropical, incluindo cerca de 25 gêneros e 300 espécies. No Brasil foram encontrados naturalmente 14 gêneros e 100 espécies, aproximadamente, principalmente na floresta amazônica, onde é um dos elementos mais característicos (SOUZA \& LORENZI, 2008). Segundo SMITH et al. (2015) a região Norte, detém 105 espécies de Lecythicadeae distribuídas em 10 gêneros, o que representa 84,1\% do total das espécies da subfamília Lecythidoideae.

As espécies de Lecythidaceae além da sua grande importância ecológica para as florestais neotropicais também possuem importância econômica para a região. Na Amazônia brasileira um dos produtos florestais não madeireiros mais importantes economicamente é a castanha-do-pará, que move o extrativismo da região. As madeiras dessas espécies também são muito valorizadas na região amazônica, principalmente no estado do Pará onde atualmente são colhidas 28 espécies de Lecythidaceae para produção de madeira (SEMA-PA, 2012).

Muitos trabalhos vêm sendo desenvolvidos em torno das espécies de Lecythidaceae nas áreas de dinâmica florestal, identificação e morfologia de espécies, potencial madeireiro, regeneração natural e produtos florestais não madeireiros dentre eles destacam-se REIS et al. (2014a), VIANA \& JARDIM (2013), ROCEK et al. (2014), SMITH et al. (2016) e REIS et al. (2016). Segundo SILVA et al. (1977) há diversas espécies de Lecytidaceae arbóreas amazônicas, em geral de Eschweilera, denominadas pelo nome vulgar de mata-matá ou matamatá, invariavelmente espécies madeireiras.

No ano de 2015 foram colhidas no estado do Pará 11 espécies de Eschweilera sendo retirado um volume de $30.132,99 \mathrm{~m}^{3} . \mathrm{ha}^{-1}$, representando $1,5 \%$ do volume total de madeiras colhidas no estado (SEMMA-PA, 2015). A caracterização das estruturas anatômicas pode auxiliar na identificação correta da espécie, além de revelar características específicas que possam levar uma espécie a ser utilizada para diversos fins (REIS et al, 2014b). É comum em inventários diagnósticos a identificação por nome vulgar o que leva a futuros erros, haja vista, que uma única espécie pode ser conhecida por diversos nomes vulgares dependendo da região. Por causa desses erros de identificação pode ocorre a superexploração de espécies de madeira, ou seja, a identificação errada pode intensificar a exploração de uma determinada espécie que está agrupada com outras espécies, e a sua capacidade de suporte, como não conhecido, pode ser bem menor em uma determinada intensidade de exploração. 
Para que não ocorra a superexploração de espécies é necessário que seja realizada a identificação correta das espécies colhidas. Nesse sentido a anatomia da madeira pode auxiliar como mais uma ferramenta de identificação. Também é de fundamental importância a criação de banco de dados e armazenamento de amostras de madeiras para que se possa consultar quando houver dúvidas em relação à identificação de espécies, muitos estudos vêm colaborando com esse objetivo dentre eles destacam-se SIEGLOCH et al. (2013) e SOARES et al. (2013), GUMA et al. (2015) e MACHADO et al. (2015), todos esses trabalhos apresentam informações significativas sobre a identificação de madeiras.

A caracterização anatômica da madeira também pode fornecer informações essenciais para a indústria manufatureira sobre aspectos importantes de espécies comerciais. Coleções de madeira especialmente para os trópicos fornecer uma riqueza de material de estudo ajudando investigações de capacidade de suporte dos ecossistemas. Como tal a caracterização anatômica da madeira tem fundamentos excelentes para contribuir na pesquisa em mudanças globais (BEECKMAN, 2016).

O objetivo desse estudo foi caracterizar anatomicamente através da microscopia, quatro espécies amazônicas de Eschweilera Mart. ex DC., disponíveis na xiloteca do Museu Paraense Emilio Goeldi (MPEG), para fornecer informações sobre as características dessas madeiras para a indústria manufatureira e/ou agentes que a utilizam na sua produção.

\section{MATERIAL E MÉTODOS}

O material lenhoso utilizado para a caracterização anatômica foi obtido na coleção da Xiloteca Walter A. Egler, do MPEG (Tabela 1). As espécies estudadas foram selecionadas tendo como critério a importância comercial da madeira, ou seja, a sua procura no mercado madeireiro. Para uma identificação segura e precisa, foram utilizadas amostras de madeira com material botânico correspondente no herbário destas instituições.

TABELA 1. Espécies de Eschweilera Mart. ex DC. (Lecythidaceae) da Amazônia caracterizadas anatomicamente.

\begin{tabular}{|c|c|c|c|c|}
\hline Espécie & $\begin{array}{l}\text { Nome } \\
\text { vulgar }\end{array}$ & Registro & Coletor & $\begin{array}{l}\text { Número } \\
\text { da coleta }\end{array}$ \\
\hline Eschweilera & & & & \\
\hline $\begin{array}{l}\text { amazonica } \mathrm{R} . \\
\text { Knuth }\end{array}$ & Matamatá-ci & X 1589 & Santos, M. R. & 43 \\
\hline $\begin{array}{c}\text { Eschweilera } \\
\text { coriacea (DC.) S.A. } \\
\text { Mori }\end{array}$ & $\begin{array}{c}\text { Matamatá- } \\
\text { branco }\end{array}$ & X 5670 & $\begin{array}{c}\text { Salomão, R.; } \\
\text { Rosa, N. A. \& } \\
\text { Rosário, C. }\end{array}$ & 165 \\
\hline $\begin{array}{c}\text { Eschweilera } \\
\text { juruensis R. Knuth }\end{array}$ & Mata-matá & X 5343 & Silva, N. T. & 5330 \\
\hline $\begin{array}{l}\text { Eschweilera ovata } \\
\text { (Cambess.) Miers }\end{array}$ & Mata-matá & $\times 6173$ & $\begin{array}{l}\text { Rosário, C. \& } \\
\text { Cardoso, O. N. }\end{array}$ & 979 \\
\hline
\end{tabular}




\section{Corpos de prova}

Foram selecionadas amostras de madeira da xiloteca e foram retirados corpos de prova com dimensões aproximadas de $1,5 \mathrm{~cm}$ na direção tangencial, $2 \mathrm{~cm}$ na direção radial e $3 \mathrm{~cm}$ na direção longitudinal, seguindo as orientações do Laboratório de Produtos Florestais (LPF).

\section{Caracterização macroscópica}

Os corpos de prova foram aplainados no plano transversal, longitudinal tangencial e longitudinal radial, com um micrótomo de deslize Reichter, para melhor visualização e interpretação das estruturas anatômicas da madeira se utilizou uma lupa conta-fios de 10x de aumento. Nesta fase, além da análise macroscópica, foram caracterizadas as propriedades organolépticas como: cor, textura, grã, desenho, dureza, etc.

As normas utilizadas para a descrição das estruturas anatômicas macroscópicas da madeira foram as do Instituto Brasileiro do Meio Ambiente e Recursos Renováveis/IBAMA (1992). O estudo foi conduzido no laboratório de Anatomia da Madeira do Museu Paraense Emilio Goeldi (MPEG). O registro digital fotográfico da macroscópica foi realizado na superfície transversal das amostras de madeira, com uma lupa acoplada ao microcomputador, com auxílio do Solftware Motic 2.0.

\section{RESULTADOS E DISCUSSÃO}

- Eschweilera amazonica R. Knuth

- Nome vulgar: Matamatá-ci

Informações gerais: o cerne apresenta a cor amarelo-escuro, com diferenciação entre cerne e alburno, apresenta brilho moderado. Dura ao corte manual, grã direita, textura grossa. Densidade básica da madeira de $0,90 \mathrm{~g} . \mathrm{cm}^{3}$ (FEARSIDE, 1997). Usos: as madeiras das espécies de Eschweilera por apresentarem densidade média à alta geralmente são indicadas para usos na construção civil, como pontes, postes, dormente etc.

Descrição macroscópica (Figura 1A): Parênquima axial perceptível apenas sob lente 10x, paratraqueal vasicêntrico e em linhas. Raios perceptíveis apenas sob lente de 10x nas seções transversal e tangencial; linhas vasculares irregulares na seção tangencial, não estratificados, espelhado pouco contrastado na seção radial. Poros perceptíveis apenas sob lente de 10 vezes, em arranjo diagonal, aparentemente na maioria solitários, ocorrendo também múltiplos de dois. Camadas de crescimento indistintas. 

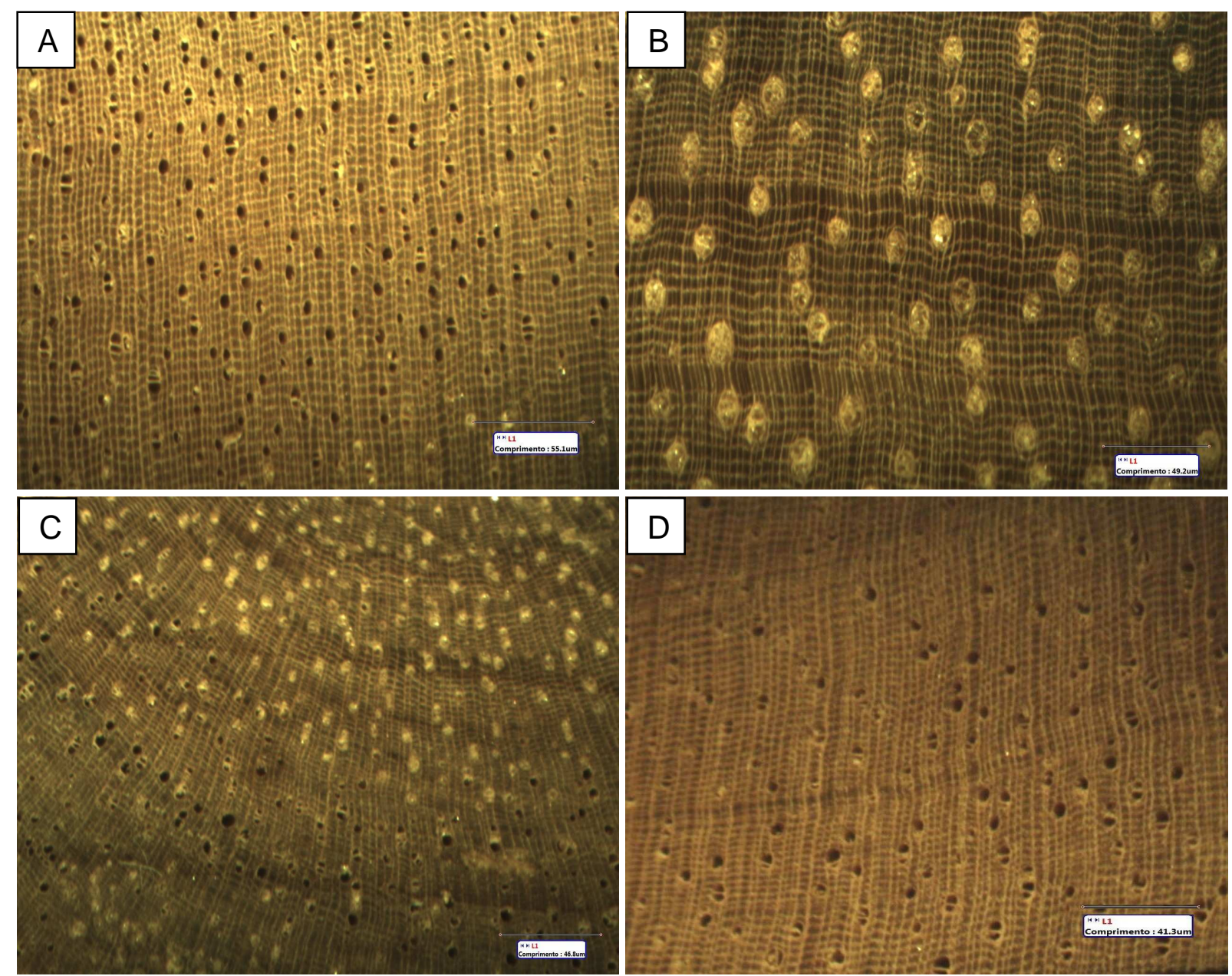

FIGURA 1: Corte transversal das espécies de Eschweilera: A - E. amazonica R. Knuth; B - E. juruensis R. Knuth.; C - E. ovata (Cambess.) Miers; D E. coriacea (DC.) S.A. Mori.

- Eschweilera juruensis R. Knuth

- Nome vulgar: Mata-matá

Informações gerais: o cerne apresenta a cor marrom-escura, sem diferenciação entre cerne e alburno, apresenta alto brilho nas superfícies longitudinais. Dura ao corte manual, grã direita, textura grossa. Densidade básica de $0,96 \mathrm{~g} \cdot \mathrm{cm}^{3}$ (ZANNE et al, 2009).

Descrição macroscópica (Figura 1B): Parênquima axial perceptíveis apenas sob lente de 10x, paratraqueal vasicêntrico e em linhas. Raios perceptíveis apenas sob lente de 10x nas seções transversal e tangencial; linhas vasculares irregulares na seção tangencial, não estratificados, espelhado contrastado na seção radial. Poros perceptíveis apenas sob lente de 10 vezes, em arranjo diagonal, aparentemente maioria solitários, ocorrendo também múltiplos de dois e totais ou parcialmente obstruídos por tilos. Camadas de crescimento indistintas.

- Eschweilera ovata (Cambess.) Miers

- Nome vulgar: Mata-matá-jibóia

Informações gerais: o cerne apresenta a cor amarelo-escuro, com diferenciação entre cerne e alburno, apresenta brilho moderado nas superfícies ENCICLOPÉDIA BIOSFERA, Centro Científico Conhecer - Goiânia, v.13 n.24; p.180 2016 
longitudinais. Dura ao corte manual, grã direita, textura grossa. Densidade básica da madeira de $0,81 \mathrm{~g} \cdot \mathrm{cm}^{3}$ (MADEIRAS, 1988).

Descrição macroscópica (Figura 1C): Parênquima axial perceptíveis apenas sob lente 10x, paratraqueal vasicêntrico e em linhas. Raios perceptíveis apenas sob lente de 10x nas seções transversal e tangencial; linhas vasculares irregulares na seção tangencial, não estratificados, espelhado contrastado na seção radial. Poros pouco perceptíveis sob lente de 10 vezes, sem arranjo, aparentemente maioria solitários, ocorrendo também múltiplos de dois, três e quatro e obstruídos por tilos. Camadas de crescimento individualizadas por zonas tangenciais mais escuras.

- Eschweilera coriacea (DC.) S.A. Mori

- Nome vulgar: Matamatá-branco, biribá, castanheira-das-águas.

Informações gerais: Cerne/alburno distintos pela cor, cerne amarronzado a oliváceo. Madeira sem brilho nas superfícies longitudinais, com cheiro imperceptível, dura ao corte transversal manual, grã entrecruzada ou revessa, textura média, figura ausente. Densidade básica da madeira de 0,73 g.cm ${ }^{3}$ (CORADIN et al, 2010). Usos mais comuns segundo PEREIRA (2013): estruturas (tesouras, vigas, pilares, pontes, caibros, ripas, estacas e cruzetas); assoalhos (rodapés, tacos, tábuas, escadas); esquadrias (portas, janelas, caixilhos, venezianas).

Descrição macroscópica (Figura 1D): Parênquima axial perceptível apenas sob lente de 10x, paratraqueal vasicêntrico e em linhas. Raios perceptíveis apenas sob lente de 10x nas seções transversal e tangencial; linhas vasculares retilíneas na seção tangencial, não estratificados, espelhado pouco contrastado na seção radial. Poros perceptíveis apenas sob lente de 10 vezes, em arranjo diagonal, aparentemente maioria solitários, ocorrendo também múltiplos de dois, três e quatro. Camadas de crescimento indistintas.

LOPES et al. (1983), PAULA (2003) e CORADIN et al. (2010) apresentaram resultados semelhantes a este estudo na descrição macroscópica de espécies do gênero Eschweilera como vasos/poros solitários, parênquima axial e raios perceptíveis apenas sob lente e parênquima axial em linhas.

O parênquima axial para a fisiologia do vegetal tem a função de armazenamento de substâncias nutritivas no lenho e é muito importante também para a identificação de espécies, pois quando visualizada a face transversal de uma madeira pode-se observar seus arranjos e distribuição, e a sua descrição é muito utilizada na diferenciação de madeiras. Foi comum em todas as espécies estudadas os tipos de parênquima paratraqueal vasicêntrico e em linhas (quadro 1). Segundo BURGER \& RICHTER (1991) em uma mesma espécie podem coexistir dois ou mais tipos de parênquima. 
QUADRO 1. Dados qualitativos das espécies estudadas.

\begin{tabular}{|c|c|c|c|c|c|}
\hline Espécie & $\begin{array}{c}\text { Arranjo } \\
\text { dos } \\
\text { poros }\end{array}$ & $\begin{array}{c}\text { Vasos/poro } \\
\mathrm{s}\end{array}$ & $\begin{array}{l}\text { Parênquima } \\
\text { axial }\end{array}$ & Raios & $\begin{array}{l}\text { Camadas de } \\
\text { crescimento }\end{array}$ \\
\hline $\begin{array}{l}\text { Eschweilera } \\
\text { amazonica } \\
\text { R. Knuth }\end{array}$ & Diagonal & Solitários & $\begin{array}{l}\text { Paratraqueal } \\
\text { vasicêntrico } \\
\text { e em linhas }\end{array}$ & $\begin{array}{l}\text { Não } \\
\text { estratifica } \\
\text { dos }\end{array}$ & Indistintas \\
\hline $\begin{array}{c}\text { Eschweilera } \\
\text { coriacea } \\
\text { (DC.) S.A. } \\
\text { Mori }\end{array}$ & Diagonal & Solitários & $\begin{array}{l}\text { Paratraqueal } \\
\text { vasicêntrico } \\
\text { e em linhas }\end{array}$ & $\begin{array}{c}\text { Não } \\
\text { estratifica } \\
\text { dos }\end{array}$ & Indistintas \\
\hline $\begin{array}{c}\text { Eschweilera } \\
\text { juruensis R. } \\
\text { Knuth }\end{array}$ & Diagonal & Solitários & $\begin{array}{l}\text { Paratraqueal } \\
\text { vasicêntrico } \\
\text { e em linhas }\end{array}$ & $\begin{array}{l}\text { Não } \\
\text { estratifica } \\
\text { dos }\end{array}$ & Indistintas \\
\hline $\begin{array}{c}\text { Eschweilera } \\
\text { ovata } \\
\text { (Cambess.) } \\
\text { Miers }\end{array}$ & $\begin{array}{l}\text { Sem } \\
\text { arranjo }\end{array}$ & Solitários & $\begin{array}{l}\text { Paratraqueal } \\
\text { vasicêntrico } \\
\text { e em linhas }\end{array}$ & $\begin{array}{l}\text { Não } \\
\text { estratifica } \\
\text { dos }\end{array}$ & $\begin{array}{l}\text { Individualiza } \\
\text { das por } \\
\text { zonas } \\
\text { tangenciais } \\
\text { mais escuras }\end{array}$ \\
\hline
\end{tabular}

Todas as espécies apresentaram poros em sua maioria solitários, de acordo com BURGER \& RICHTER (1991) e REIS et al. (2014b), os vasos (distribuição, abundância, tamanho e agrupamento) são características importantes para a identificação de espécies e propriedades tecnológicas da madeira. As espécies também apresentaram raios não estratificados. Segundo BURGER \& RICHTER (1991) estas estruturas desempenham as funções de armazenamento, transporte e condução transversal de substâncias nutritivas, apresentando uma grande riqueza morfológica, variando em tipo, número e tamanho de células, sendo juntamente com o parênquima axial os mais eficazes elementos de distinção entre espécies.

Eschweilera ovata (Cambess.) Miers foi a espécie que mais se diferenciou das outras do gênero por não apresentar arranjo dos poros e principalmente por apresentar camadas de crescimento individualizadas por zonas tangenciais mais escuras. As camadas de crescimento são boas ferramentas para a identificação de espécies. Em muitas árvores tropicais, a camada de crescimento corresponde a períodos de chuva e períodos de seca, queda das folhas e/ou simplesmente dormência (BURGER \& RICHTER, 1991; REIS et al., 2014b).

\section{CONCLUSÕES}

A caracterização anatômica das madeiras de Eschweilera permite as seguintes conclusões:

- Apenas Eschweilera ovata diferencia-se das outras espécies do gênero por apresentar camadas de crescimento visivelmente nítidas na macroscópia.

- As espécies de Eschweilera deste estudo apresentaram muitas características semelhantes, sendo assim, indicado à identificação microscópica para melhor conhecimento de suas estruturas. 


\section{REFERÊNCIAS}

BEECKMAN, H. Wood Anatomy and Trait-Based ecology. IAWA Journal, v. 37, n. 2, p. 127-151, 2016. Disponível em: < http://dx.doi.org/10.1163/22941932-20160127>. doi: 10.1163/22941932-20160127

BURGER, L. M.; RICHTER, H. G. Anatomia da madeira. 154p. Nobel, Barueri, 1990.

CORADIN,V. T. R.; CAMARGOS, J. A. A.; PASTORE, T. C. M.; CHRISTO, A. G. Madeiras comerciais do Brasil: chave interativa de identificação baseada em caracteres gerais e macroscópicos $=$ Brazilian commercial timbers: interactive identification key based on general and macroscopic features. Serviço Florestal Brasileiro, Laboratório de Produtos Florestais: Brasília, 2010. CD-ROM.

DADZIE, P. K.; AMOAH, M.; FRIMPONG-MENSAH, K.; OHENEBA-KWARTENG, F. Some physical, mechanical and anatomical characteristics of stemwood and branchwood of two hardwood species used for structural applications. Materials and Structures, v. 49, n. 12, p. 4947-4958, 2016. Disponível em: <http://dx.doi.org/10.1617/s11527-016-0835-3>. doi: 10.1617/s11527-016-0835-3

FEARNSIDE, P. Wood density for estimating forest biomass in Brazilian Amazonia. Forest Ecology and Management, Amsterdã, v. 90, n. 1, p. 59-87, 1997.

GUMA, R.; BALDIN, T.; DENARI, L.; HASELEIN, C. R.; MARCHIORI, J. N. C. Anatomia da madeira de Tetrorchidium rubrivenium Poepp. \& Endl. (Euphorbiaceae), Balduinia, n. 47, p. 12-18, 2015. Disponível em: <http://dx.doi.org/10.5902/2358198017229>. doi: 10.5902/2358198017229

INSTITUTO BRASILEIRO DO MEIO AMBIENTE E DOS RECURSOS NATURAIS RENOVÁVEIs (IBAMA). Normas parar procedimntos em estudos de anato mia de madeira: I.angiospermae, II.gimnospermae. Brasília: 19 p. (Série Técnica, 15) 1992.

LOPES, C. A. C.; PINTO, R, G.; DOURADO, R. S. A.; PERES, A. S. G.; BRANDÃO, A. T. O. Propriedades físico-mecânicas e usos comuns de espécies de madeiras da Amazônia. Belém, SUDAM, 1983. 97 p.

MACHADO, P. F. S.; MARCHIORI, J. N. C.; SIEGLOCH, A. M. Anatomia da madeira de Enterolobium glaziovii (Benth.) Mesquita. Balduinia, n. 50, p. 11-15, 2015. Disponível em: <http://dx.doi.org/10.5902/235819820324>. doi: $10.5902 / 235819820324$

MADEIRAS da Amazônia: características e utilização: Estação Experimental de Curuá- Una. Brasília, DF: Conselho Nacional de Desenvolvimento Científico e Tecnológico: Instituto Brasileiro de Desenvolvimento Florestal, Laboratório de Produtos Florestais, 1988. v. 2, 236 p. 
PAULA, J. E. Caracterização anatômica da madeira de sete espécies da Amazônia com vistas à produção de energia e papel. Acta Amazonica, v. 33, n. 2, p.243-262, 2003. Disponível em: <http://dx.doi.org/10.1590/1809-4392200332262>. doi: $10.1590 / 1809-4392200332262$

PEREIRA, A. F. Madeiras Brasileiras - Guia de combinação e substituição. FAPEMIG. 1. ed. São Paulo: Editora Blucher, 2013. 130p.

REIS, P. C. M.; REIS, L. P.; RUSCHEL, A. R.; SILVA, J. N. M.; CARVALHO, J. O. P.; QUEIROZ, W. T. Effect of timber harvesting on density and basal area of Lecythidaceae species in the Eastern Amazon. Floresta, v.44, n. 2, p.229 - 238, 2014(a). Disponível em: <http://dx.doi.org/10.5380/rf.v44i2.33043>. doi: 10.5380/rf.v44i2.33043

REIS, P. C. M.; REIS, A. R. S.; LISBOA, P. L. B.; REIS, L. P. Anatomia do lenho de seis espécies do gênero Cecropia Loefl. (Urticaceae). Boletim do Museu Paraense Emílio Goeldi. Ciências Naturais, v. 9, n. 1, p. 169-181, 2014(b). Disponível em: <http://www.museu-goeldi.br/editora/bn/artigos/cnv9n1_2014/anatomia(reis).pdf>.

REIS, P. C. M.; RUSCHEL, A. R.; REIS, L. P.; SOARES, M. H. M.; CRUZ, E. D. Lecythidaceae encontradas na floresta de Moju, Pará. Belém: Embrapa - CPATU, Documentos 416.33 p. 2016.

ROCEK, J. M.; GILBERT, M. E.; BROADBENT, E. N. Brazil Nut (Bertholletia excelsa, Lecythidaceae) Regeneration in Logging Gaps in the Peruvian Amazon. International Journal of Forestry Research. v. 2014, n. ID 420764, p. 1-8, 2014. Disponível em: <http://dx.doi.org/10.1155/2014/420764>. doi: 10.1155/2014/420764

SECRETARIA DE ESTADO DE MEIO AMBIENTE (SEMA-PA). Extração e Comércio de Toras de Madeira Nativa por Essência: 2015. Disponível em:<http://monitoramento.sema.pa.gov.br/sisflora/index.php/relatorios >. Acesso em: 20/08/2016.

SECRETARIA DE ESTADO DE MEIO AMBIENTE (SEMA-PA). Extração e Movimentação de Toras de Madeira Nativa: 2006 a 2012. Disponível em:< http://monitoramento.sema.pa.gov.br/sisflora/>. Acesso em: 16/10/2012. Acesso em: 16/10/2012.

SIEGLOCH, A. M.; MARCHIORI, J. N. C.; SANTOS, S. R. Anatomia da madeira de dezesseis espécies de Euphorbiaceae lato sensu, com base na análise de agrupamento de caracteres anatômicos. Balduinia, n. 40, p. 09-17, 2013. Disponível em: <http://dx.doi.org/10.5902/23581980>. doi: 10.5902/23581980

SILVA, M. F. LISBOA, P. L. B.; LISBOA, R. C. L. Nomes Vulgares de Plantas Amazônicas. Manaus, INPA, p. 211, 1977.

SMITH, N. P.; MORI, S. A.; LAW, W.; RIBEIRO, M. Conservation assessment of Lecythidaceae from eastern Brazil. Kew Bulletin, 71, p. 1-19, 2016. Disponível em: <http://dx.doi.org/10.1007/S12225-016-9627-8>. doi: 10.1007/S12225-016-9627-8 
SMITH, N.P.; MORI, S.A.; PRANCE, G.T. Lecythidaceae: Lista de Espécies da Flora do Brasil. Jardim Botânico do Rio de Janeiro. Disponível em: <http://floradobrasil.jbrj.gov.br/jabot/floradobrasil/FB145>. Acesso em: 30/12/2015.

SOARES, W. F.; MELO, L. E. L.; LISBOA, P. L. B. Anatomia do Lenho de Cinco Espécies Comercializadas como 'sucupira', Floresta e Ambiente, v. 21, n. 1, p. 114125, 2013. Disponível em: <http://dx.doi.org/10.4322/floram.2013.042>. doi:10.4322/floram.2013.042

SOUZA, V.; LORENZI, H. Botânica Sistemática: guia ilustrado para identificação das famílias Fanerógamas nativas e exóticas no Brasil, baseado em APG II. 2 ed. Nova Odessa, São Paulo: Instituto Plantarum, 2008.

VIANA, A. C. N.; JARDIM, F. C. S. Natural regeneration dynamics of Couratari guianensis in a tropical forest selectively logged in Moju, state of Para, Brazil. Revista de Ciências Agrárias, v. 56, n. 2, p. 112-119, 2013. Disponível em: <http://dx.doi.org/10.4322/rca.2013.018>. doi: 10.4322/rca.2013.018

ZANNE, A. E.; LOPEZ-GONZALEZ, G.; COOMES, D. A.; ILIC, J.; JANSEN, S.; LEWIS, S. L.; MILLER, R. B.; SWENSON, N. G.; WIEMANN, M. C.; CHAVE, J. Global wood density database. Durham: Dryad, 2009. Disponível em: <http://hdl.handle.net/10255/dryad.235>. Acesso em: 06/02/2015. 